\title{
FOTODEGRADAÇÃO HETEROGÊNEA DE EFLUENTE DE POLPA KRAFT BRANQUEADA COM CLORO ${ }^{1}$
}

\begin{abstract}
Geraldo de Aguiar Coelho², Regina Weinschutz³, Mário José Dallavalli³ e Álvaro Luiz Mathias³
RESUMO - A polpa celulósica da madeira deve ser branqueada e alvejada para atingir elevado valor comercial. O cloro é muito eficiente no branqueamento da polpa obtida por processo químico, mas também produz subprodutos altamente poluentes e tóxicos. Outros produtos químicos de branqueamento têm sido propostos para reduzir a toxicidade, mas são mais caros e menos versáteis. A fotodegradação catalítica com o uso de radiação ultravioleta (UV) foi feita para avaliar o potencial de redução do teor de poluentes encontrados em um efluente de branqueamento de celulose com o uso de cloro. A polpa celulósica de Pinus taeda foi produzida e branqueada em laboratório. Três litros de efluente receberam $3 \mathrm{~g}$ de dióxido de titânio $\left(\mathrm{TiO}_{2}\right)$ e foram submetidos à radiação UV. Essa radiação foi produzida com o uso da ampola de lâmpada de vapor de mercúrio de alta pressão de $250 \mathrm{~W}$ (12.500 Lm) durante 240 min. A fotodegradação a $25^{\circ} \mathrm{C}$ por 240 min com UV reduziu em cerca de $43 \%$ a cor, $40 \%$ a $\mathrm{DBO}_{5}, 50 \%$ a DQO, $40 \%$ de material calcinável e $45 \%$ do carbono orgânico total, além de baixar a toxicidade LC $_{50}$ para o efluente tratado. O bioindicador Artemia salina apresentou o tempo de sobrevida maior do que $1.000 \%$ para o efluente tratado. A distribuição dos pontos experimentais de 0 a 240 min sugere que a fotodegradação deveria ser estendida. Assim, a redução de poluentes observada sugere que esse processo oxidativo avançado (POA), com fotocatálise com o uso de $\mathrm{UV}$ e $\mathrm{TiO}_{2}$, pode ser conveniente para o tratamento de efluentes clorados de branqueamento. Isso pode viabilizar o uso seguro de branqueamento com cloro.
\end{abstract}

Palavras-chave: Pinus taeda; Fotocatálise; Oxidação de efluente.

\section{HETEROGENEOUS PHOTODEGRADATION OF EFFLUENT FROM KRAFT PULP BLEACHED WITH CHLORINE}

\begin{abstract}
The wood cellulose pulp must be bleached to reach a high commercial value. Chlorine is very effective in bleaching for pulp obtained by chemical process, but it also produces highly polluting and toxic byproducts. Other bleaching chemicals have been proposed to reduce toxicity, but they are more expensive and less versatile. Catalytic photodegradation using ultraviolet radiation (UV) was performed to assess the potential for reducing the content of pollutants in an effluent from bleaching cellulose pulp by using chlorine. The Pinus taeda cellulose pulp was produced and bleached in laboratory. Three liters of effluent received $3 \mathrm{~g}$ of titanium dioxide (TiO2) and were submitted to UV radiation. UV was produced with the use of a mercuryvapor bulb in high-pressure of $250 \mathrm{~W}(12,500 \mathrm{Lm})$ for $240 \mathrm{~min}$. Photodegradation to $25^{\circ} \mathrm{C}$ for $240 \mathrm{~min}$ with UV decreased about $43 \%$ of the color, $40 \%$ of the BOD5 (biochemical oxygen demand), $50 \%$ of the COD (chemical oxygen demand), $40 \%$ of calcinable material and $45 \%$ of TOC (total organic carbon), in addition to decreasing the toxicity LC50 (lethal concentration - 50\%) for the treated effluent. The bioindicator Artemia salina showed the survival time greater than 1,000\% for the treated effluent. The experimental points distribution from 0 to 240 min suggests that the photodegradation must be extended. Thus, the reduction of pollutants observed suggests that advanced oxidation process (AOP), using UV-TiO2 photocatalytic oxidation may be convenient to treat chlorinated bleaching effluents. This can enable the safe use of chlorine bleaching.
\end{abstract}

Keywords: Pinus taeda; Photocatalysis; Effluent oxidation.

\footnotetext{
${ }^{1}$ Recebido em 26.09.2012 aceito para publicação em 24.07.2014.

${ }^{2}$ Serviço Nacional de Aprendizagem Industrial, Centro Técnico em Celulose e Papel, Brasil. E-mail: <geraldo.coelho@pr.senai.br> .

${ }^{3}$ Departamento de Engenharia Química, Universidade Federal do Paraná, Brasil. E-mail: <reginawz@ufpr.br>,<dalla@ufpr.br> e $<$ mathias@ufpr.br>.
} 


\section{INTRODUÇÃO}

A composição da madeira pode ser descrita como água, extrativos, celulose, hemiceluloses, lignina e os elementos inorgânicos ( $\mathrm{Ca}, \mathrm{Na}, \mathrm{Mg}, \mathrm{Mn}, \mathrm{Fe}$, K etc.). A produção da polpa de celulose pode ser realizada por diversos processos industriais. Para o processo kraft, os cavacos de madeira são cozidos em uma solução de hidróxido de sódio e sulfeto de sódio. O material solubilizado contém a maior parte da lignina e de fragmentos de hemiceluloses da madeira, bem como alguns fragmentos de celulose. Mesmo assim, a fração residual de lignina presente na polpa bruta a mantém com cor bege a marrom, pois contém muitos radicais cromóforos. Assim, as fibras de celulose precisam ser branqueadas com cozimento pelo uso de outros agentes químicos. Cerca de $90 \%$ da lignina residual do processo de cozimento, além de fragmentos de carboidratos, é degradada pelos produtos químicos aplicados durante os estágios de branqueamento (YEBER et al., 2000). Esse rendimento pode variar de acordo com as concentrações dos reagentes, as temperaturas e os tempos de residência em cada estágio do branqueamento (CARDOSO, 2006; DENCE; REEVE, 1996). Segundo Hise (1996), nenhum outro produto químico pode atingir o desempenho da cloração com tanta economia. Entretanto, essa tecnologia gera um efluente que potencialmente contém quantidades relevantes de clorolignina, ou seja, cloro ligado a compostos fenólicos. Dessa forma, esse efluente dificilmente poderá ser tratado de maneira eficiente em estações de tratamentos biológicos convencionais (BERTAZZOLI; PELEGRINI, 2002). No entanto, as fábricas que possuem sequência de branqueamento ECF (livres de cloro elementar) também apresentam compostos organoclorados em lodos gerados em suas Estações de Tratamento de Efluentes, o que é atribuído à presença de dióxido de cloro (GUERRA et al., 2012).

Processos oxidativos avançados com base na geração de espécies reativas, incluindo radicais hidroxilas obtidos por processo fotoquímico (KARCI, 2014) ou fotoeletroquímico (BERTAZZOLI; PELEGRINI, 2002), são relatados como opções viáveis na destruição de contaminantes orgânicos refratários em efluentes industriais, como clorofenóis. Mesmo assim, a toxicidade dos produtos gerados igualmente deve ser avaliada. Assim, a Artemia salina, um invertebrado aquático, tem sido usada para esse tipo de abordagem (KARCI, 2014).
O objetivo deste trabalho foi avaliar a redução da cor, da carga orgânica e da toxicidade do efluente de branqueamento de polpa de celulose kraft de Pinus taeda L. com o uso de fotodegradação catalítica, além de radiação ultravioleta e dióxido de titânio.

\section{MATERIAL E MÉTODOS}

Cavacos de madeira de Pinus taeda L. com 16 anos de idade foram fornecidos por uma empresa da região do Município de Telêmaco Borba, no Estado do Paraná. Os cavacos com 25 a 30 mm de comprimento foram selecionados manualmente, com o auxílio de paquímetro, e cozidos em laboratório para produzir polpa celulósica do tipo kraft. A solução de digestão kraft foi produzida com 240 g de hidróxido de sódio e $150 \mathrm{~g}$ de sulfeto de sódio nona-hidratado, o que permitiu obter um teor de álcali de $15 \%\left(\mathrm{Na}_{2} \mathrm{O}\right)$. Os cavacos (1.120,3 g, em base seca) foram misturados com a solução de digestão na proporção de $1 \mathrm{~kg}$ para $4.000 \mathrm{~mL}$. A mistura foi aquecida a $170^{\circ} \mathrm{C}$ em digestor rotativo REGMED (AU 20, BRASIL), modificado para quatro câmaras de cozimento e em condições similares à industrial. A temperatura foi avaliada a cada $2 \mathrm{~min}$, sendo realizado alívio de pressão (degasagem) até $90{ }^{\circ} \mathrm{C}$, conciliando a remoção do gás atmosférico e pouca perda de componentes mais voláteis do licor. A temperatura foi progressivamente aumentada e depois mantida a $170{ }^{\circ} \mathrm{C}$ até o final do cozimento. O valor do fator $\mathrm{H}$ desejado nesse cozimento era de 2.900, o qual foi calculado pela integral da curva da velocidade de reação em função do tempo (ASSUMPÇÃO et al., 1988; SIXTA; RUTKOWSKA, 2007). Assim, o tempo de reação foi interrompido em $294 \mathrm{~min}$. O sistema foi resfriado a $40{ }^{\circ} \mathrm{C}$ e a mistura de reação, filtrada em peneira de 200 mesh. A polpa foi filtrada e o licor preto, descartado. A polpa foi lavada com água corrente sobre a peneira para retirar o máximo de licor preto residual. A polpa resultante foi desagregada em desagregador laboratorial REGMED (DSG 2000, BRASIL) durante $10 \mathrm{~min}$. O material foi depurado em depurador Laboratorial TMI (Valley Flat Screen, EUA) e ficou sobre a placa com ranhuras do depurador, sendo assim considerado como "rejeito". A polpa aprovada foi coletada em peneira de 200 mesh e considerada como polpa de celulose kraft depurada. A soma dessa polpa depurada e do rejeito foi considerada "rendimento bruto". 
Os métodos usados para caracterizar as soluções, cavacos, polpa de celulose e efluentes foram (ABNT, 1985a) NBR 9382 (determinação de matéria orgânica por perda por calcinação - duplicata) (ABNT, 1985b), NBR 9383 (umidade do cavaco de madeira - duplicata) (ABNT, 1997a), NBR 14001 (extração de compostos da madeira (ceras e graxas) em extrator Soxhlet, com o uso de diclorometano - determinar os extrativos duplicata) (ABNT, 1997b), NBR 14003 (concentração de fibras celulósicas contida em meio aquoso consistência da pasta de celulose - duplicata), (ABNT, 1998a), NBR 6039 (quantidade de água presente no papel ou celulose - umidade no papel - duplicata) (ABNT, 1998b), NBR 14030 (umedecimento e desagregamento de fibras celulósicas - operação normatizada de individualização das fibras - duplicata) (ABNT, 2001), NBR 2470 (papel, cartão e pastas celulósicas - medida do fator de reflectância difusa no azul-alvura ISO), Método HACH 2014 - 8000 (determinação de DQO demanda química de oxigênio duplicata), Método HACH 2014 - 8025 (cor real e aparente - 465 nm - aspectos físicos de solução aquosa - duplicata), Método HACH 2014 - 10129 (determinação de COT (carbono orgânico total da HACH - duplicata), Método HACH 2014 - DBO (Determinação de DBO - demanda bioquímica de oxigênio - duplicata), Método Reed \& Müench (concentração letal para 50\% da população LC50 - a toxicidade foi expressa em horas para que a população do bioindicador fosse reduzida a 50\% de seres vivos (REED; MÜNCH, 1938) - toxicidade), TAPPI - T 204 cm-97 (Extrativos (óleos e graxas - duplicata), TAPPI -T 278 cm-99 (Separação de rejeitos contida na polpa celulósica com o uso de depurador equipado com peneira com rasgos de 0,25 mm - fibras aglomeradas, palitos e nós) e TAPPI - T 624 cm-00 (teor de $\mathrm{NaOH}, \mathrm{Na}_{2}$ S e álcali total titulável no líquido de polpação - caracterização da solução de digestão).

A polpa depurada foi branqueada a partir de cinco etapas sucessivas: cloração, extração alcalina e três etapas de hipocloração consecutivas (processo CEH). Para cloração, a polpa foi diluída até a consistência de 1,5\%. Gás cloro (Carbocloro, BRASIL) foi borbulhado através de dispersor de vidro sinterizado na suspensão em baixa agitação. A cloração foi mantida a $\mathrm{pH}$ 1,5 e a $25^{\circ} \mathrm{C}$ por $45 \mathrm{~min}$, durante toda a aplicação de cloro. Após essa operação, a suspensão fibrosa foi filtrada em peneira de 200 mesh. A fração líquida foi guardada para compor parte do efluente de branqueamento. A polpa, por sua vez, foi lavada com $4 \mathrm{~L}$ de água corrente e, então, centrifugada para remoção de grande parte do líquido aderido. Esse líquido de lavagem também foi separado como efluente de branqueamento.

Para a extração alcalina, uma solução de hidróxido de sódio $10 \%$ foi aplicada à polpa com consistência de $10 \%$. A carga de álcali foi de $5 \mathrm{~kg}$ para cada $100 \mathrm{~kg}$ de polpa. A suspensão com pH 13 foi acondicionada em saco plástico duplo e manipulada manualmente por 15 s, para homogeneização da mistura. Os sacos plásticos foram colocados em um banho térmico a $60^{\circ} \mathrm{C}$ por 120 min. A seguir, a polpa foi filtrada em peneira (200 mesh) e lavada com $4 \mathrm{~L}$ de água corrente e centrifugada. A fração líquida foi recolhida e adicionada ao efluente de branqueamento.

Para a hipocloração, a polpa foi diluída a 10\% (m/v) na solução de oxidante. Hipoclorito de sódio comercial (Q-Boa, Brasil, teor de cloro de 2,5\%,) foi aplicado na proporção de 1,5 kg para $100 \mathrm{~kg}$ de polpa. Essa etapa foi realizada a $60^{\circ} \mathrm{C}$, por $180 \mathrm{~min}$. A polpa foi centrifugada e o líquido, recolhido para compor o efluente de branqueamento. As duas etapas subsequentes de hipocloração foram repetidas nas mesmas condições do primeiro estágio de hipocloração. Após o terceiro estágio, a polpa sobre a peneira (200 mesh) foi lavada até atingir $\mathrm{pH}$ próximo a neutro. $\mathrm{O}$ filtrado foi recolhido e adicionado ao efluente de branqueamento. A polpa foi centrifugada e o líquido desprendido, recolhido para, da mesma forma, compor o efluente. Assim, todas as frações líquidas das etapas de branqueamento formaram uma mistura (37.000 mL), denominada efluente de branqueamento equalizado, para produzir o fenômeno de equalização, similarmente ao que a indústria faz.

A experiência de fotocatálise foi realizada em uma câmera fechada. Uma lâmpada de vapor de mercúrio de alta pressão OSRAM (HQL250, 250 W, 12500 Lm, Brasil) teve seu bulbo removido para ser usada como fonte de radiação ultravioleta (UV). Uma fração de efluente de branqueamento equalizado (3.000 mL) recebeu dióxido de titânio (3 g) e foi submetido à fotodegradação em um Becker (4.000 mL) apoiado em um agitador magnético. A fonte de radiação UV ficou a $30 \mathrm{~cm}$ da superfície, sendo ela reajustada conforme se retiravam as alíquotas (150 mL) de amostras. A mistura reagente foi agitada durante todo o período de fotodegradação e mantida cerca de $25^{\circ} \mathrm{C}$ por $4 \mathrm{~h}$ de radiação. Todas as amostras foram avaliadas em duplicatas. Amostras do efluente equalizado depois da reação fotocatalítica foram retiradas para análises de diversos parâmetros, e notou-se que

Revista Árvore, Viçosa-MG, v.38, n.5, p.927-934, 2014 
os pontos que descrevem um modelo empírico são valores médios de duplicatas. O coeficiente de variação $(\mathrm{CV})$ foi usado para análise de medida de dispersão central, o qual é considerado como baixo, quando inferior a 10\%; médio, quando está entre 10 e 20\%; alto, quando aferido entre 20 e 30\%; e muito alto, quando superior a $30 \%$ (GOMES, 1990).

\section{RESULTADOS}

O licor de polpação, os cavacos e a polpa celulósica bruta produzida foram caracterizados para alguns de seus parâmetros (Tabela 1). Nesse sentido, diversos parâmetros ambientais do efluente de branqueamento equalizado foram determinados (Tabela 2). O efeito do tratamento fotocatalítico catalisado $\left(\mathrm{UV}-\mathrm{TiO}_{2}\right)$ foi avaliado a partir da redução de cor (Figura 1), de carga orgânica (Figura 2) e de toxicidade (Figura 3). A média do coeficiente de variação foi de $2 \%$ para $\mathrm{pH}, 3 \%$ para DQO, 3\% para COT, $10 \%$ para $\mathrm{DBO}_{5}, 12 \%$ para cor e $12 \%$ para materiais calcináveis, sendo, portanto, baixo (<10\%) ou médio (entre 10 e 20\%) (GOMES, 1990).

\section{DISCUSSÃO}

A polpa celulósica kraft e seu efluente de branqueamento equalizado foram produzidos em laboratório, de modo que não houvesse contaminação

Tabela 1 - Características do licor branco para o cozimento do cavaco de Pinus taeda e da polpa kraft.

Table 1 - Characteristics of white liquor for the cooking of wood chips of Pinus taeda and Kraft pulp.

\begin{tabular}{|c|c|c|c|c|c|}
\hline \multicolumn{2}{|c|}{ Licor } & \multicolumn{2}{|c|}{ Cavaco } & \multicolumn{2}{|l|}{ Polpa } \\
\hline $\begin{array}{c}\text { Álcali ativo } \# 1 \\
\left(\text { g.L }{ }^{-1} \text { em } \mathrm{Na}_{2} \mathrm{O}\right)\end{array}$ & $104,20 \pm 0,51$ & Umidade (\%) & $49,25 \pm 1,25$ & Rendimento bruto (\%) & $43,5 \pm 0,93$ \\
\hline $\begin{array}{l}\text { Álcali efetivo } \# 2 \\
\left(\text { g. } L^{-1} \text { em } \mathrm{Na}_{2} \mathrm{O}\right)\end{array}$ & $96,71 \pm 0,51$ & $\begin{array}{c}\text { Extrativos totais } \\
\text { (\%, base seca) }\end{array}$ & $3,84 \pm 0,30$ & Rendimento depurado (\%) & $42,8 \pm 0,87$ \\
\hline $\begin{array}{c}\text { Álcali total } \\
\text { titulável } \\
\left.\text { (g. } \mathrm{L}^{-1} \text { em } \mathrm{Na}_{2} \mathrm{O}\right)\end{array}$ & $104,20 \pm 0,50$ & Lignina Klason & $26,36 \pm 0,43$ & Teor de rejeito (\%) & $0,7 \pm 0,02$ \\
\hline Sulfidez ${ }^{\# 4}(\%)$ & $14,37 \pm 1,62$ & $\begin{array}{l}\text { Holocelulose } \\
\text { (\%, base seca) }\end{array}$ & $70,82 \pm 1,39$ & Número Kappa & $28,09 \pm 1,32$ \\
\hline
\end{tabular}

Tabela 2 - Características iniciais do efluente do branqueamento.

Table 2 - Initial characteristics of the effluent from bleaching.

\begin{tabular}{|c|c|c|c|}
\hline Parâmetro & & Parâmetro & \\
\hline $\begin{array}{c}\text { Cor (Absorbância em } 465 \text { nm) } \\
\left.\text { (mg Pt-Co.L }{ }^{-1}\right)\end{array}$ & $12,33 \pm 0,09$ & $\mathrm{DBO}_{5} / \mathrm{DQO}$ & $0,17 \pm 0,02$ \\
\hline $\mathrm{pH}$ & $8,52 \pm 0,11$ & Calcináveis (\%) & $1072,00 \pm 0,94$ \\
\hline $\mathrm{DBO}_{5}\left(\mathrm{mg} \cdot \mathrm{L}^{-1}\right)$ & $206,00 \pm 21$ & Carbono orgânico total (mg. $\mathrm{L}^{-1}$ ) & $1073,5 \pm 43,5$ \\
\hline DQO $\left(\mathrm{mg} \cdot \mathrm{L}^{-1}\right)$ & $1189,75 \pm 24$ & Toxicidade (h) & $<1 \pm 0,0$ \\
\hline
\end{tabular}

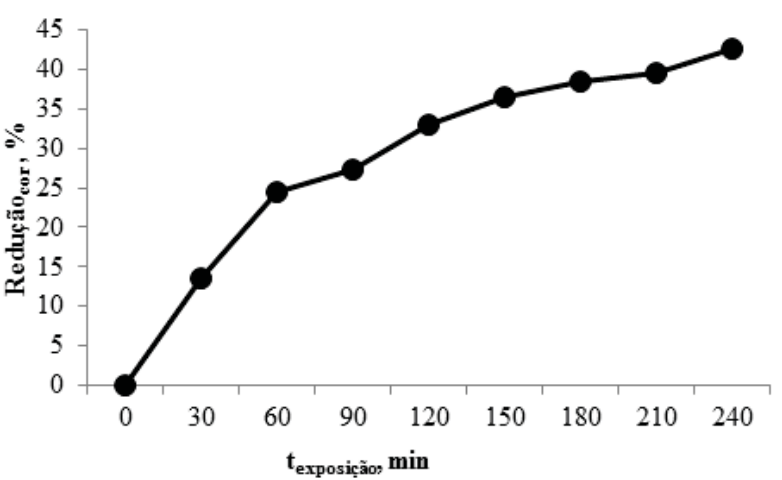

Figura 1 - Redução de cor ao longo do tempo de fotodegradação. Figure 1 - Reduction in color over time of photodegradation.

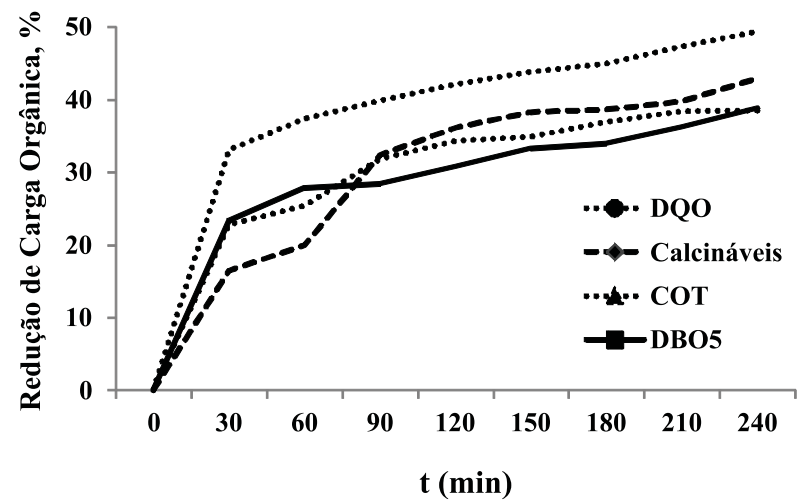

Figura 2-Redução de carga orgânica (DQO, matérias calcináveis, $\mathrm{COT}_{\text {e }} \mathrm{DBO}_{5}$ ) ao longo do tempo de fotodegradação.

Figure 2-Reduction of organic load (COD, calcinable material, TOC and $\mathrm{BOD}_{5}$ ) over time of photodegradation.

Revista Árvore, Viçosa-MG, v.38, n.5, p.927-934, 2014 


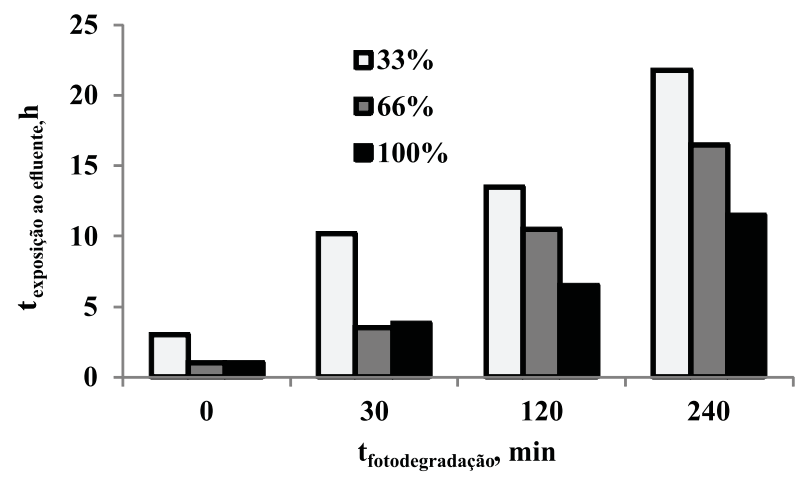

Figura 3 - Redução de toxicidade ao longo do tempo de fotodegradação para efluente não diluído (100\%) e diluído a $66 \%$ ou 33\% (tempo de exposição maior para sobrevida de 50\% de indivíduos de Artemia salina significa menor toxicidade).

Figure 3-Reduction of the toxicity over time of photodegradation for effluent undiluted (100\%) and diluted to 66\% or $33 \%$ (longer exposure time for survival of $50 \%$ of the individuals of Artemia salina means lower toxicity).

com outros produtos químicos ou efluentes de outras fontes. O teor de álcali ativo e o teor de álcali efetivo (Tabela 1) são valores típicos para o processo Kraft (ASSUMPÇÃO et al., 1988; BIERMANN, 1996; CARDOSO, 2006). A solução foi preparada com reagentes com elevado grau de pureza; por exemplo, sem a típica ocorrência da carbonatação do hidróxido de sódio; logo, a diferença entre o álcali total titulável e álcali ativo foi nula (ASSUMPÇÃO et al., 1988). A sulfidez até $15 \%$, sendo $14,4 \%$ a observada na Tabela 1 , favorece a deslignificação da polpa e está dentro da realidade industrial (KLOCK, 2008).

Os teores de macrocomponentes dos cavacos revelaram elevado teor de lignina insolúvel em ácido dessa madeira (Tabela 1). A quantidade de cavacos e da solução de digestão usada gerou polpa com rendimento compatível com o observado na indústria (CARDOSO, 2006). A depuração eliminou apenas 0,7\% de matéria, e o número Kappa (28,1, Tabela 1) é compatível com valores relatados para pinus (MAIA; COLODETTE, 2003) e com a ordem de grandeza verificada industrialmente (CARDOSO, 2006). Assim, a metodologia laboratorial produziu uma polpa kraft com parâmetros semelhantes àqueles observados na industrial e com a vantagem de ocorrer em condições controladas.

O fator H da polpação foi 2900, o qual representa o efeito do tempo e da temperatura de cozimento e é condizente com a situação real. Esse parâmetro é essencial, pois valores de fator $\mathrm{H}$ semelhantes, mesmo que tenha havido diferença na combinação de temperatura e de tempo, devem resultar em polpas com características similares, isto é, com a deslignificação próxima (ASSUMPÇÃO et al., 1988). Ao final do cozimento, foram obtidos $4.400 \mathrm{~mL}$ de licor preto e 535,7 g de polpa. O rendimento da polpa bruta está dentro da grandeza esperada. Ainda, o baixo valor encontrado para o rejeito revelou a alta eficiência da metodologia empregada. O número Kappa abaixo de 30 revelou que a lignina presente nas fibras pode ser facilmente eliminada (VASCONCELOS, 2005). Diante do exposto, a polpa usada no branqueamento simula a realidade industrial, mas com a peculiaridade de poder ser reproduzida, inclusive, sem apresentar interferentes do meio técnico.

Após as cinco etapas de branqueamento, a polpa apresentou elevada alvura $(89,0 \% \pm 0,0)$, compatível com os limites industriais, que são de cerca de $90 \%$ (SILVA, 2010). O rendimento da polpa branqueada (85,27\%) revelou que $14,73 \%$ da polpa depurada foi solubilizada. Assim, a fração mássica perdida indica que a carga de materiais orgânicos presentes no efluente equalizado é muito elevada, o que é comprovado pelos altos valores de DQO, materiais calcináveis e carbono orgânico total (Tabela 2). É pressuposto que o material solubilizado contém aproximadamente $90 \%$ da lignina residual do processo de cozimento, além de hemiceluloses e celuloses degradadas pelos produtos químicos aplicados durante os estágios de branqueamento (YEBER et al., 2000). Esse rendimento pode variar segundo a concentração dos reagentes, a temperatura e o tempo de residência em cada estágio do branqueamento (DENCE; REEVE, 1996; CARDOSO, 2006). Mesmo assim, é indiscutível que a carga orgânica é elevada e há a presença de expressivo teor de compostos cromóforos (Tabela 2). Isso pode ser observado principalmente na etapa de extração alcalina, em que os compostos coloridos da polpa celulósica foram solubilizados no álcali após o estágio oxidante da cloração. Tal fato confirma a afirmação de Hise (1996) de que nenhum outro produto químico pode atingir seu desempenho da cloração com tamanha economia. O pH resultante dos efluentes depois de equalizado era alcalino ( $\mathrm{pH} 8,5)$, pois, embora a cloração seja em condição ácida ( $\mathrm{pH}$ 1,5), o pH é aumentado depois de ser combinado; os demais efluentes, no caso dos estágios de extração alcalina ( $\mathrm{pH} 13)$ e de hipocloração (pH 11).

Revista Árvore, Viçosa-MG, v.38, n.5, p.927-934, 2014 
Os valores de materiais calcináveis (1.072 mg.L ${ }^{-1}$ ) e COT (1073,5 mg. $\mathrm{L}^{-1}$, Tabela 2) estão associados a uma elevada concentração de compostos orgânicos. A DBO inicial do efluente era de $206 \mathrm{mg} . \mathrm{L}^{-1}$. Balcioglu et al. (2007) relataram valores com cerca de 120 mg. $\mathrm{L}^{-1}$ no efluente de branqueamento convencional, enquanto Morais (2006) e Yeber (2000) descreveram valores que podem chegar a $534 \mathrm{mg}$. $\mathrm{L}^{-1} \mathrm{em}$ branqueamentos tipo ECF (livre de cloro elementar). Os valores de DBO (206 mg. $\mathrm{L}^{-1}$ ) e de DQO (1.190 mg.L $\mathrm{L}^{-1}$, Tabela 2) são compatíveis com valores de DBO, entre 200 e 800 mg. $\mathrm{L}^{-1}$; e DQO entre 500 e 1.500 mg. $L^{-1}$, descritos por Pereira (2007). Essa informação também demonstra a considerável variação na grandeza desses parâmetros nos efluentes de branqueamentos de fábricas de celulose. Bertazzoli e Pelegrini (2002) informaram valores semelhantes de DQO para efluentes de branqueamento no estágio de extração alcalina. A relação DBO/DQO é frequentemente utilizada para verificar a biodegradabilidade de um efluente. O valor encontrado $(0,17)$ é muito menor do que o limite mínimo $(0,30)$ para ser considerado biodegradável (MORAIS et al., 2006). Esse fato pode ser associado à presença de cloro-lignina no efluente de branqueamento com cloro elementar. Tal efluente dificilmente poderá ser tratado em estações de tratamentos biológicos convencionais (BERTAZZOLI; PELEGRINI, 2002), mas, a título de solução da questão, sistemas biológicos diferenciados têm sido propostos, a exemplo do de fitorremediação (GUERRA et al., 2012). A elevada toxicidade do efluente foi confirmada pelo baixo valor atribuído a LC $_{50}$ (Tabela 2); e o tempo de sobrevivência de 50\% dos espécimes do bioindicador foi inferior a $1 \mathrm{~h}$, quando exposto ao efluente de branqueamento equalizado.

A fotodegradação catalítica foi capaz de reduzir a cor do efluente (Figura 1). A descoloração foi mais intensa durante os 120 min iniciais da reação e atingiu o valor de $43 \%$ para 240 min. Portanto, a tendência de comportamento dos pontos experimentais sugere que a fotodegradação deveria ser prolongada para atenuar esse aspecto negativo.

A fotodegradação de 240 min apresentou redução de $\mathrm{DBO}_{5}$ (206 mg. $\mathrm{L}^{-1}$ ) na ordem de 40\% (Figura 2). A DQO teve seu valor da inicial (1.189,75 mg. $\mathrm{L}^{-1}$ ) reduzido a quase 50\% (602,4 mg. $\left.\mathrm{L}^{-1}\right)$. A redução da DQO foi proporcionalmente mais intensa nos primeiros $60 \mathrm{~min}$, o que sugere que existem moléculas com diferentes potenciais para serem fotodegradadas. Assim, a relação
$\mathrm{DBO}_{5} / \mathrm{DQO}$ inicial $(0,17)$ foi acrescida até 0,20 após $30 \mathrm{~min}$ de fotodegradação e mostrou leve tendência de aumento caso a reação se prolongasse. Mesmo assim, ela está distante de ser superior ou igual a 0,3, para que seja considerada biodegradável (KARRER et al., 1997; YEBER et al., 2000; MORAIS et al., 2006).

A fotodegradação reduziu o teor de materiais calcináveis na ordem de $40 \%$ e o carbono orgânico total (COT), em 38\% (Figura 2). A propensão à degradação persistiria caso o tempo de tratamento fosse prolongado. A redução de COT de 35\% por fotodegradação também foi narrada para efluente da $1^{\text {a }}$ extração alcalina, o que seria melhor que a eficiência de lodo ativado, que é capaz de reduzir entre 10 e 30\% a matéria orgânica em 20 dias (BERTAZZOLI; PELEGRINI, 2002). Logo, a fotodegradação é muito mais rápida e eficiente e deve ser considerada em futuros projetos para implantação de uma planta de tratamento de efluentes.

A tolerância do bioindicador Artemia salina foi elevada de $<1 \mathrm{~h}$ para $3 \mathrm{~h}$ quando o efluente de branqueamento equalizado foi diluído a $66 \%$ ou $33 \%$ de sua concentração inicial. Isso pode explicar por que foi praticado descarte desse rejeito sem a percepção de danos ambientais até poucos anos atrás. A fotodegradação, portanto, foi capaz de reduzir a toxicidade, o que pode representar não só a destruição de alguns componentes presentes no efluente, mas também que seus subprodutos são menos tóxicos. Após 240 min de fotodegradação, o bioindicador pode resistir a um tempo de exposição de $1.100 \%$ superior ao efluente não tratado.

\section{CONCLUSÃO}

A fotodegradação de efluente do processo de branqueamento CEH com o uso de radiação UV na presença de $0,1 \mathrm{~g} \%$ de $\mathrm{TiO}_{2}$ por 240 min reduziu em cerca de $43 \%$ a cor, $40 \%$ a DBO, $50 \%$ a DQO, $40 \%$ os materiais calcináveis e 45\% a COT, além de ter diminuído a toxicidade. Existe, ainda, a tendência de que a fotodegradação pode ser maior para tempos mais prolongados de reação. A degradação é mais intensa no início, quando o efluente é mais concentrado ou tem mais moléculas suscetíveis a ela. O efluente tratado por fotodegradação pode ser considerado menos tóxico. Assim, a redução de poluentes observada sugere que esse processo oxidativo avançado (POA) com fotocatálise e uso de radiação ultravioleta em presença de dióxido 
de titânio pode ser conveniente para o tratamento rápido de efluentes clorados de branqueamento. Aparentemente, processos consecutivos de fotorremediação ou biológicos especiais de lagoas poderiam produzir o polimento final do efluente. Caso isso se confirme, o processo de branqueamento com o emprego de cloro poderia ser novamente utilizado em virtude de sua grande flexibilidade, mas sem causar impactos ambientais relevantes.

\section{AGRADECIMENTOS}

A UFPR, SENAI, FUNDAÇÃO ARAUCÁRIA, FUNPAR, CAPES e CNPq, pelo apoio.

\section{REFERÊNCIAS}

\section{ASSOCIAÇÃO BRASILEIRA DE NORMAS} TÉCNICAS - ABNT. NBR 9382: Produtos orgânicos e inorgânicos - Determinação da perda por calcinação. Rio de Janeiro: 1985a.

ASSOCIAÇÃO BRASILEIRA DE NORMAS TÉCNICAS - ABNT. NBR 9383: Produtos orgânicos sólidos - Determinação da umidade e outros materiais voláteis nas condições do teste. Rio de Janeiro: 1985b.

ASSOCIAÇÃO BRASILEIRA DE NORMAS TÉCNICAS - ABNT. NBR 14001: Pastas celulósicas - Determinação de material solúvel em diclorometano. Rio de Janeiro: 1997a.

\section{ASSOCIAÇÃO BRASILEIRA DE NORMAS} TÉCNICAS - ABNT. NBR 14003: Pastas celulósicas - Determinação da consistência. Rio de Janeiro: 1997b.

\section{ASSOCIAÇÃO BRASILEIRA DE NORMAS} TÉCNICAS - ABNT. NBR 6039: Papel, cartão e papelão - Determinação da umidade por secagem em estufa. Rio de Janeiro, 1998a.

\section{ASSOCIAÇÃO BRASILEIRA DE NORMAS} TÉCNICAS - ABNT. NBR 14030: Pastas celulósicas - Desagregação a úmido em laboratório. Rio de Janeiro: 1998b.

\section{ASSOCIAÇÃO BRASILEIRA DE NORMAS} TÉCNICAS - ABNT. NBR 2470: Papel, cartão e pastas celulósicas - Medida do fator de reflectância difusa no azul (alvura ISO). Rio de Janeiro: 2001.
ASSUMPÇÃO, R. M. et al. Polpação química. In: SENAI/IPT Celulose e Papel - Tecnologia de fabricação da pasta celulósica. São Paulo: Escola SENAI “Theobaldo de Nigris”, 1988. 964p.

BALCIOGLU, A. et al. Merits of ozonation and catalytic ozonation pre-treatment in the algal. Journal of Environmental Management, v.85, n.4, p.918-929, 2007.

BERTAZZOLI, R.; PELEGRINI, R. Descoloração e degradação de poluentes orgânicos em soluções aquosas através do processo fotoeletroquímico. Química Nova, v.25, n.3, p.477-482, 2002.

BIERMANN, C. J. Pulping Fundamentals. In: BIERMANN, C. J. Handbook of pulping and papermaking. 2.ed. San Diego: Academic Press, 1996. v.1. p.55-100.

CARDOSO, G. S. Fabricação de celulose. Curitiba: SENAI, 2006. v.1.349p.

DENCE, C. W.; REEVE, D. W. Pulp bleaching - principles and practice. Atlanta: Tappi Press, 1996. 880p.

GOMES, F. P. Curso de estatística experimental. 12. ed. São Paulo: Nobel, 1990. 467p.

GUERRA, M. A. S. L. et al. Avaliação da utilização de composto orgânico produzido a partir dos resíduos de fábrica de celulose quanto aos níveis de organoclorados e metais. Revista Árvore, v.36, n.2, p.381-387, 2012.

HISE, R. Chlorination. In: DENCE, C. W.; REEVE, D. W. Pulp bleaching - principles and practice. Atlanta: Tappi Press, 1996. 880p.

KARCI, A. Degradation of chlorophenols and alkylphenol ethoxylates, two representative textile chemicals, in water by advanced oxidation processes: the state of the art on transformation products and toxicity. Chemosphere, v.99, n.1, p.1-18, 2014.

KARRER, N. J.; RYHINER, G.; HEINZLE, E. Applicability test for combined biologicalchemical treatment of wastewaters containing biorefractory compounds. Water Research, v.31, n.5, p.1013-1020, 1997.

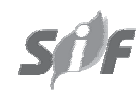


KLOCK, H. Curso de Engenharia Industrial Madeireira. Curitiba: Universidade Federal do Paraná, 2008. 9p.

MORAIS, A. A. Uso de ozônio como pré e pós-tratamento de efluentes da indústria de celulose kraft branqueada. 2006. 96f. Dissertação (Mestrado em Engenharia Civil). Universidade Federal de Viçosa, Viçosa, MG, 2006.

MAIA, E. P., COLODETTE, J. L. Efeito do conteúdo e da natureza da lignina residual na eficiência e na seletividade do branqueamento com ozônio. Revista Árvore, v.27, n.2, p.217232, 2003.

MORAIS, J. L. D.; SIRTORI, C.; PERALTAZAMORA, P. G. Tratamento de chorume de aterro sanitário por fotocatálise heterogênea. Química Nova, v.29, n.1, p.20-23, 2006.

HACH. Method 8000. http://www.hach.com. Metodologia disponiblizada pelo fornecedor. Acesso em: 4 de ago. de 2014.

HACH. Method 8025. http://www.hach.com. Metodologia disponiblizada pelo fornecedor. Acesso em: 4 de agosto de 2014.

HACH. Method 10129. http://www.hach.com. Metodologia disponiblizada pelo fornecedor. Acesso em: 4 de ago. de 2014.

HACH. Method DBO. http://www.hach.com. Metodologia disponiblizada pelo fornecedor. Acesso em: 4 de ago. de 2014.

PEREIRA, R. D. O. Combinação de tratamento biológico aeróbio com processos oxidativos avançados visando intensificar a remoção de matéria orgânica em efluentes da indústria têxtil e de celulose Kraft. 2007. 100f. Dissertação (Mestrado em Engenharia Civil) Universidade Federal de Viçosa, Viçosa, MG, 2007.
REED, L. J.; MÜNCH, H. A simple method of estimating fifth percent endpoints. American Journal of Hygiene, v.27, n.3, p.495-497, 1938.

SILVA, D. D. J. Química da parte úmida em processo de fabricação de papel interações em interfaces sólido-líquido. 2010. 325f. Tese (Doutorado em engenharia química) - Escola Politécnica da Universidade de São Paulo, São Paulo, 2010.

SIXTA, H.; RUTKOWSKA E. W. Estudo cinético abrangente da polpação kraft de Eucalyptus globulus. O Papel, v.73 , n.4, p. 68-81, 2007.

TECHNICAL ASSOCIATION FOR THE WOLDWIDE PULP, PAPER AND CONVERTING INDUSTRY - TAPPI. Standard test methods. Preparation of wood for chemical analysis - T204 cm-97. Atlanta: Tappi Press, 1997.

TECHNICAL ASSOCIATION FOR THE WOLDWIDE PULP, PAPER AND CONVERTING INDUSTRY - TAPPI. Standard test methods. Pulp screening (Valley-type screening device)-T278cm-99. Atlanta: Tappi Press, 1999.

TECHNICALASSOCIATION FOR THE WOLDWIDE PULP, PAPER AND CONVERTING INDUSTRY - TAPPI. Standard test methods. Analysis of Soda and Sulfate White and Green Liquors - T624 cm-00. Atlanta: Tappi Press, 2000.

VASCONCELOS, F. S. R. D. Avaliação do processo SuperBatch ${ }^{\mathrm{TM}}$ de polpação de Pinus taeda. 2005. 106f. Dissertação (Mestrado em Recursos Florestais) - Universidade de São Paulo - Escola Superior de Agricultura "Luiz de Queiroz”, Piracicaba, 2005.

YEBER, M. C. et al. Photocatalytic degradation of cellulose bleaching effluent by supported $\mathrm{TiO}_{2}$ and ZnO. Chemosphere, v.41, n.8, p.1193-1197, 2000. 